\title{
Investigation of a Short-Range Underwater Acoustic Communication Channel for MAC Protocol Design
}

\author{
Gunilla E. Burrowes \\ School of Electrical Engineering and Computer Science \\ University of Newcastle \\ Newcastle, Australia \\ Email: gunilla@ieee.org
}

\author{
Jamil Y. Khan \\ School of Electrical Engineering and Computer Science \\ University of Newcastle \\ Newcastle, Australia \\ Email: jamil.khan@newcastle.edu.au
}

\begin{abstract}
Mobile swarms of autonomous underwater vehicles (AUV) have exciting potential for extending current AUV applications and adding new possibilities to the working environment of the oceans. For swarm operations to occur, fast reliable communication between all vehicles within the swarm is needed.

The challenge for underwater communication is the very different and unique characteristics of the channel compared to the terrestrial environment. Acoustic communication has been the typical physical layer technology used for underwater operations and has experienced significant research and progress over the last few decades, however this focus has been predominately on longer range acoustic channels.

The work presented in this paper is to determine the critical parameters of an underwater acoustic communication channel for the evaluation and development of a new MAC Protocol designed for short range communication between vehicles operating within a swarm. This paper provides a review of the essential channel characteristics and the modelling currently in use for this purpose, highlighting the difference for short range operations. It then assesses the electro-acoustic interface with investigation into the parametric relationship between digital modulation requirements and short range channel characteristics.
\end{abstract}

\section{INTRODUCTION}

Oceans, which cover over $70 \%$ of the earth's surface, are being looked at in a new light as they become a source of future resource potential as well as a source of information about the earth's carbon ecosystem and it affect on climate change. To unlock the oceans secrets there are major projects and research being undertaken around the world to develop technologies required to operate within the oceans. The development of small autonomous underwater vehicles (AUV) has been rapid over the last decade and there are several operating now as single units [1], [2]. Swarms of these vehicles are yet to be realised as methods to communicate between each other very quickly and precisely are needed. The vehicles within swarm operations can work as closely as $10 \mathrm{~m}$ but depending on different mission requirements may operate to more than 300 to $500 \mathrm{~m}$. The requirements for collaborative communication are to avoid collisions within the swarm and with objects in the swarms path as well as to facilitate improved operations by building on the ability to operate as a team which would give a swarm of vehicles much more potential than any single vehicle working on its own.

The MAC layer protocol is a vital component for any communication system, responsible for maximising network throughput while minimising energy consumption which are critical factors for the development of a mobile swarm communication system. However to develop a MAC protocol it is necessary to understand the short range acoustic channel to leverage any potential advantages in channel utilisation.

The physical layer acoustic channel characteristics are well documented but have focused predominately on longer range communication, from $500 \mathrm{~m}$ to over $20 \mathrm{~km}$. More recent work has begun to looked at shorter range operation predominately for multi hop purposes [3], [4], [5].

The communication performance for underwater wireless links have been shown to exhibit frequency, spacial and timevarying characteristics that are strongly dependent on the variability of the underwater channels themselves, and these are presented in Section 2. Specific reference to how these characteristics vary at shorter ranges will be discussed. Section 3 then develops the sonar equations for short range propagation with an analysis of the Signal-to-Noise ratio (SNR) for short range and comparisons to longer range analysis. Section 4 evaluates the probability of bit error and date rate characteristics for varying channel and physical layer parameters. The paper concludes with identifying the critical parameters for short range underwater acoustic data communication.

\section{Underwater Acoustic Channel}

An AUV swarm network can be likened to a wireless sensor network (WSN) in many of its features including its communication functionality. AUV's are deployed as spatially distributed autonomous sensor nodes and are required to self-organise to perform collaborative monitoring and search functions as is the case for WSN. The underwater acoustic channel however has some fundamental differences to RF channel which require essentially new designs of the MAC protocol. In particular, the acoustic channel introduces a very high propagation delay which is $0.67 \mathrm{~ms} / \mathrm{m}$ (compared to RF of $3.33 \mathrm{~ns} / \mathrm{m}$ in air). RF, underwater, even at low frequencies suffers from extreme attenuation due to conductive seawater and high rates of absorption that has predominately eliminated its use for underwater communications. In addition, underwater communication channel characteristics change more dynamically than in terrestrial channels due to its attenuation, 
noise and thermal profiles [6] as will be presented in this section.

\section{A. Source Level - Transmitter Power}

An acoustic source is defined in terms of the pressure level at a distance of $1 \mathrm{~m}$ (area at $1 \mathrm{~m}$ is $12.6 \mathrm{~m}^{2}$ ). The acoustic intensity of the reference wave for sea water is: $I_{\text {ref }}=\left(P a_{\text {ref }}\right)^{2} / \rho * c\left(W m^{-2}\right)$, using a reference pressure level $\left(P a_{\text {ref }}\right)$ of $1 \mu \mathrm{Pa}$ and $\rho$ the density of the medium and $c$ the speed of sound.

$$
S L_{t x}=10 \log \left(\left(P_{t x} / 12.6 * d\right) / I_{\text {ref }}\right) \quad d B
$$

where $d=$ depth in meters, $P_{t x}$ is the total power consumed by the projector.

\section{B. Propagation Loss}

An acoustic propagating signal underwater experiences two major propagation losses; spreading and absorption. Spreading loss is due to the weakening of the sound signal as it geometrically spreads outward from the source. The absorption or attenuation loss represents predominately the effects of absorption of sound waves as they propagate outward varying linearly with range and so is expressed as $\mathrm{dB}$ per unit distance. Propagation or Transmission Loss (TL) thus can be written as follows with $\alpha(f, d, t)$ referring to the absorption coefficient.

$$
T L(r, f, d, t)=k * 10 \log (r)+\alpha(f, d, t) * r * 10^{-3}
$$

where $r$ is range in $\mathrm{m}$, and $k$ the spreading factor $(\mathrm{k}=1$ cylindrical, =2-spherical)

\section{Absorption Loss}

Extensive measurements of absorption losses over the last half century has lead to several empirical formula which take into account frequency, salinity, temperature, $\mathrm{pH}$, depth and speed of sound. A popular version is Thorp's expression [7], [8], Equation 3, which is valid for frequencies from $100 \mathrm{~Hz}$ to $1 \mathrm{MHz}$ and is based on seawater with salinity of $35 \% \mathrm{ppt}$, $\mathrm{pH}$ of 8 , temperature of $4^{\circ} \mathrm{C}$ and depth of $0 \mathrm{~m}$ (atmospheric pressure, depth is assumed but not stated by Thorp). These values are used as reference if not stated.

$$
\begin{gathered}
\alpha(f)=\frac{0.11 f^{2}}{1+f^{2}}+\frac{44 f^{2}}{4100+f^{2}}+2.75 \times 10^{-4} f^{2}+ \\
0.0033 \\
d B / k m \\
\alpha(f, d, t)=\frac{A_{1} f_{1} f^{2}}{f_{1}^{2}+f^{2}}+\frac{A_{2} P_{2} f_{2} f^{2}}{f_{2}^{2}+f^{2}}+ \\
A_{3} P_{3} f^{2} \\
d B / k m
\end{gathered}
$$

The Fisher and Simmons model, Equation 4, includes the relaxation frequencies of Boric acid and $\left(\mathrm{M}_{g} \mathrm{SO}_{4}\right)$ molecules as $f_{1}, f_{2}$ respectively, and use coefficients " $\mathrm{A}$ " to represent the effects of temperature, and "P" coefficients represent depth. These can be found in several references [7], [9], [10].
The absorption loss term is shown in Figure 1 with a comparison of the two absorption coefficient models described, and two frequency levels, and shows that $\alpha$ increases with increasing frequency at any fixed temperature and depth and increases with increasing range.

It can be seen that the Thorp model shows a conservative value for the ranges and frequencies of interest up to $300 \mathrm{~m}$. The Fisher and Simmons model for a particular frequency however provides some insight into the variations due to depth and temperature. In the region below $100 \mathrm{kHz}$, temperature change has a more significant affect on $\alpha$ than depth [11], but above these frequencies depth begins to dominate [9], [10]. Sehgal [10] showed that at higher frequencies above $300 \mathrm{kHz}$, Thorps model predicts lower losses as the relaxation frequencies, developed and introduced by Fisher and Simmons, are not included.

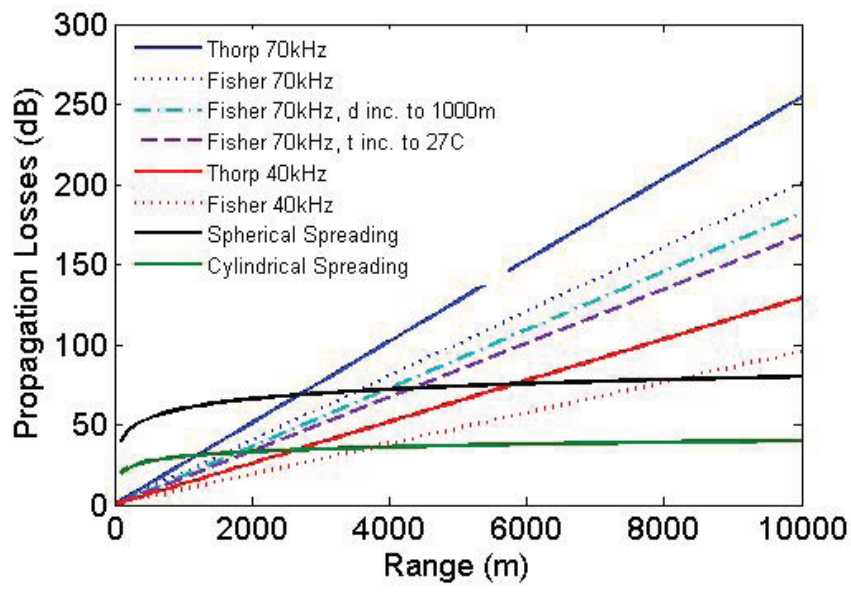

Fig. 1. Propagation Losses vs Short Ranges. Spreading and absorption factors

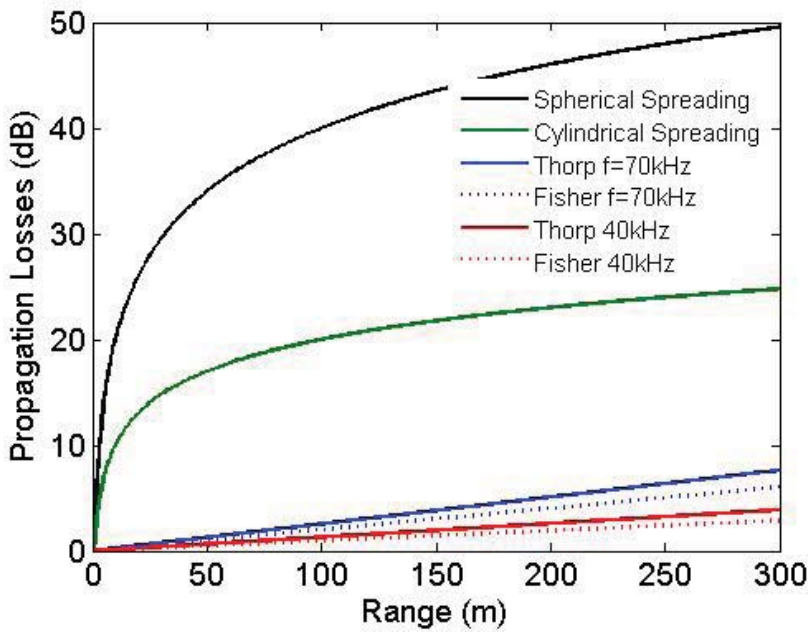

Fig. 2. Propagation Losses vs Short Ranges. Spreading and absorption factors 


\section{Transmission Loss}

Figure 1 and 2 show the propagation losses against range, for longer and shorter ranges respectively, separated into the two Transmission Loss terms, Spreading and Absorption. For very short range communication the contribution of the absorption term is less significant than the spreading term as can be seen in Figure 2. As range increases, Figure 1, the absorption term begins to dominate and therefore any variations in $\alpha$ also becomes more significant.

The two important characteristics for overall transmission loss at short range using the two models, Thorp and Fisher and Simmons, are:

- at shorter ranges, spherical spreading loss dominates over absorption loss, and thus the "k" term has a significant impact on the attenuation of the signal at very short range, and

- at a range in the order of $300 \mathrm{~m}$ the absorption loss at $70 \mathrm{kHz}$ exceeds that at $40 \mathrm{kHz}$ by approximately $3 \mathrm{~dB}$ (which can also be seen in Figure 1) and as range increases the difference also increases, effectively meaning that the communication channel is strongly band-limited and available bandwidth is a decreasing function of range.

\section{E. Noise}

There are three major contributors to noise underwater: ambient or background noise of the ocean; self noise of the vehicle and the intermittent site dependent noise. Ambient and self-noise will be investigated below with intermittent sources, such as ice cracking, biological and seismic events, not included as they are more readily able to be filtered out [12].

Ambient Noise in the ocean has been well defined [13], having a continuous power spectrum density (psd) and a Gaussian distribution of amplitude. It is made up of four components, each having a dominating influence in different portions of the frequency spectrum. In general, the ambient noise psd decreases with increasing frequency, refer to Figure 3. At a frequency over $100 \mathrm{kHz}$ (depending on wind state), the ambient thermal noise component begins to dominate and the overall noise psd begins to increase.

- Turbulence noise influences only the very low frequency regions $f<10 \mathrm{~Hz}$ : $10 \log N_{\text {turb }}(f)=17-30 \log (f)$

- Shipping noise dominates the $10-100 \mathrm{~Hz}$ region and has defined a shipping activity factor of $\mathrm{s}$ whose value ranges from 0 to 1 for low to high activity respectively: $10 \log N_{\text {ship }}(f)=40+20(s-0.5)+26 \log (f)-60 \log (f+$ $0.03)$

- Wave and other surface motion caused by wind and rain is a major factor in the mid frequency region of $100 \mathrm{~Hz}$ $-100 \mathrm{kHz}$ where wind speed is given by $\mathrm{w}$ in $\mathrm{m} / \mathrm{s}(1 \mathrm{~m} / \mathrm{s}$ is 2 knots):

$10 \log N_{\text {wind }}(f)=50+7.5 w^{1 / 2}+20 \log (f)-40 \log (f+$ $0.4)$

- Thermal noise becomes dominate over 100kHz: $10 \log N_{\text {th }}(f)=-15+20 \log (f)$ where $f$ is in $\mathrm{kHz}$.

Ambient Noise power also decreases with increasing depth as the distance from the surface and therefore shipping and wind noise becomes more distant as documented in Caruthers [14] showing noise can be $9 \mathrm{~dB}$ higher in swallow water. However as the vehicles are working in close proximity there is an expectation that there will always be a level of shipping noise associated with other vehicles in the swarm, irrespective of the operating depth, although this will have limited affect on the frequency range above $10 \mathrm{kHz}$ as can be seen in Figure 3 , with 's' the shipping factor.

Self noise is define as the noise generated by the vehicle itself as the platform for receiving signals. This noise can reach the hydrophone mounted on the AUV either through the mechanical structure or through the water passing over the hydrophone. In general, as with ambient noise, there is decreasing levels of self noise with increases in frequency but self noise is also significantly affected by speed with decreasing noise spectra when the vessels are travelling at slower speeds or are stationary [13], [15]. The self noise of different size and types of vehicles are as varied as there are vehicle designs [16]. There is very little published research on available values of self noise on underwater vehicles.

Holmes [2] at WHOI recently looked at the self noise of REMUS, a torpedo shape AUV, used as a towed array. Values as high as $130 \mathrm{~dB}$ re $1 \mu \mathrm{Pa}$ at $1 \mathrm{~m}$ directly aft of the vehicle at $\mathrm{f}=1 \mathrm{kHz}$ and moving a 3 knots were recorded. AUV's would typically radiate less noise in free operating conditions compared with tethered conditions. Also, with the potential slower speed operations in a swarm and higher communication system frequencies there is significant questions as to how much self noise will be representative of realistic operating conditions.

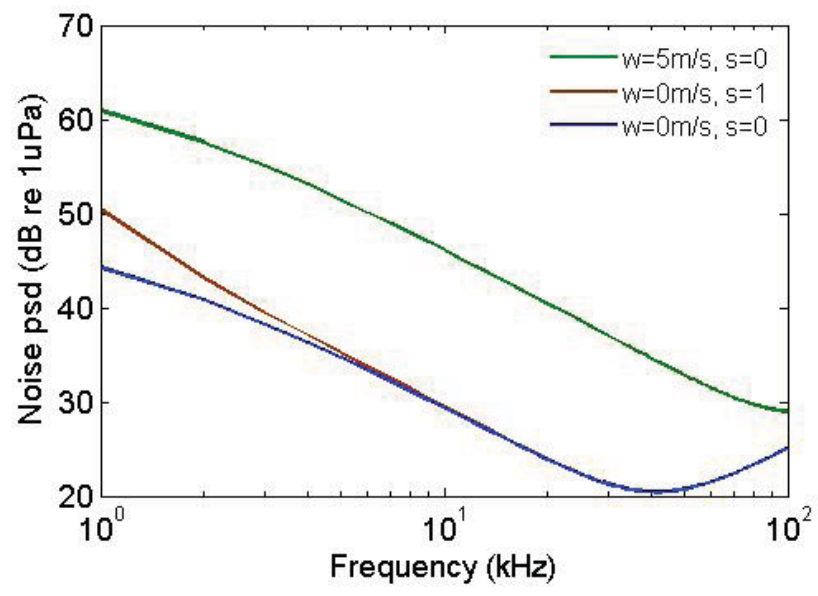

Fig. 3. Power Spectral density of the Ambient Noise

\section{F. Multi-path}

For digital communication systems, multi-path is another form of propagation loss through fading of the signal and can also cause inter-symbol interference (ISI) at the receiver which 
is a significant issue for acoustic underwater communication due to the latency of propagation [17]. There are two major mechanisms responsible for creating these multi-paths and these are reverberation (reflections and scattering) and ray bending [13], [18], [19]. Multi-path formation is different in deep and shallow water. Shallow water being dominated by reverberation whereas deep water by ray bending.

These differences are reflected in the breath of multipath models. More recent work is attempting to use statistical methods to characterize this time variant channel [20], [21], [22]. Alternative work has classified the deep underwater channel to be Ricean fading, since the sea surface and sea floor represent fixed scatterers in the acoustic medium. However, experiments show that the behaviour of the channel depending on the conditions can be either a Ricean or a Rayleigh channel. In conditions with stronger winds and shallower waters, the channel behaves as a Rayleigh fading channel. The shallower the channel and closer to the surf zones generally the poorer the performance [9]. Fading and ISI are not considered in this piece of work but will be included when more complex specific channel conditions are considered and time, frequency and spacial dynamics are taken into account.

\section{Acoustic Channel Model}

The dynamics and variability of parameters underwater means that there is no one distinctive acoustic environment and therefore no 'typical' underwater acoustic communication channel [6], and therefore no 'one' model to fit all scenarios. Thus the need for comparatively simple equations representing the average sound field, while retaining dependence upon the principal features of the environment, is appropriate for first order approximations of a system response and the passive sonar equations have served this purpose [13], [19], [23], [24].

\section{A. Sonar Equations}

The acoustic link budget uses basic sonar theory to estimate the signal to noise ratio at the receiver. The source level is determined by;

$$
S L_{t x}\left(P_{t x}\right)=170.8+10 \log P_{t x}+10 \log \eta_{t x}+D I_{t x} \quad d B
$$

where $P_{t x}$ represents the electrical power supplied to the antenna; $\eta_{t x}$ is the electro-acoustic efficiency of the projector; and DI the directivity index representing the gain in the projector. Projector and hydrophone directivity can significantly influence the number of signal paths and therefore is very important [17] to investigate, however in this work DI is assumed to be $0 \mathrm{~dB}$ representing omni-directional transceivers.

The received signal level $S L_{r x}$ based on a single path value is thus:

$$
\begin{aligned}
S L_{r x}\left(r, f, d, t, P_{t x}\right)= & S L_{t x}\left(P_{t x}\right)-T L(r, f, d, t)+ \\
& 10 \log \eta_{r x}+D I_{r x}
\end{aligned}
$$

Therefore, the Signal-to-Noise-Ratio (SNR) observed at the receiver over a range $r$ is evaluated using the noise characteristics found above.

$$
S N R\left(r, f, P_{t x}, w, s, \text { selfnoise, } B\right)=\frac{S L_{r x}\left(r, f, d, t, P_{t x}\right)}{\sum N(f, w, s, \text { selfnoise }) * B}
$$

where $\mathrm{B}$ is the receiver bandwidth

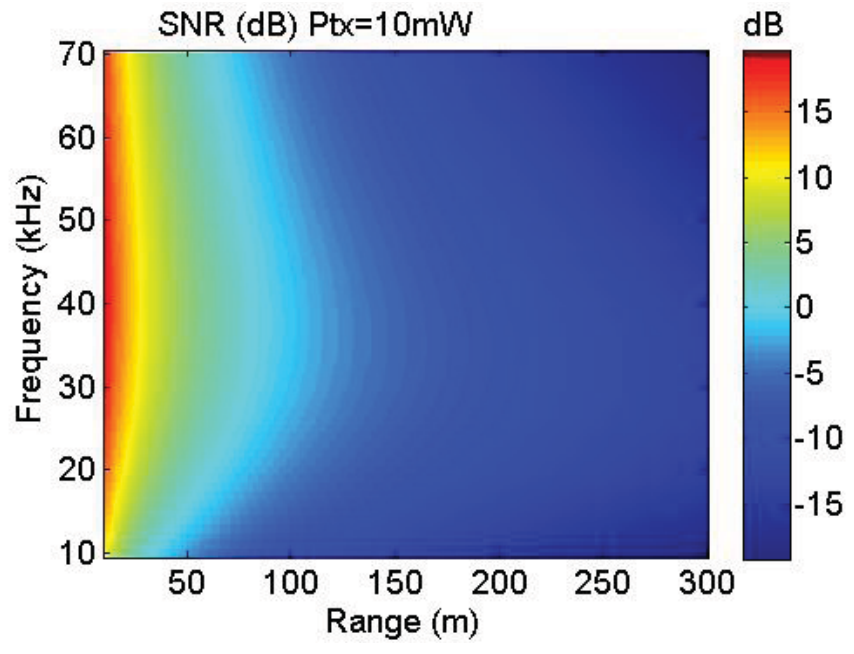

Fig. 4. SNR Ptx $=10 \mathrm{~mW}$

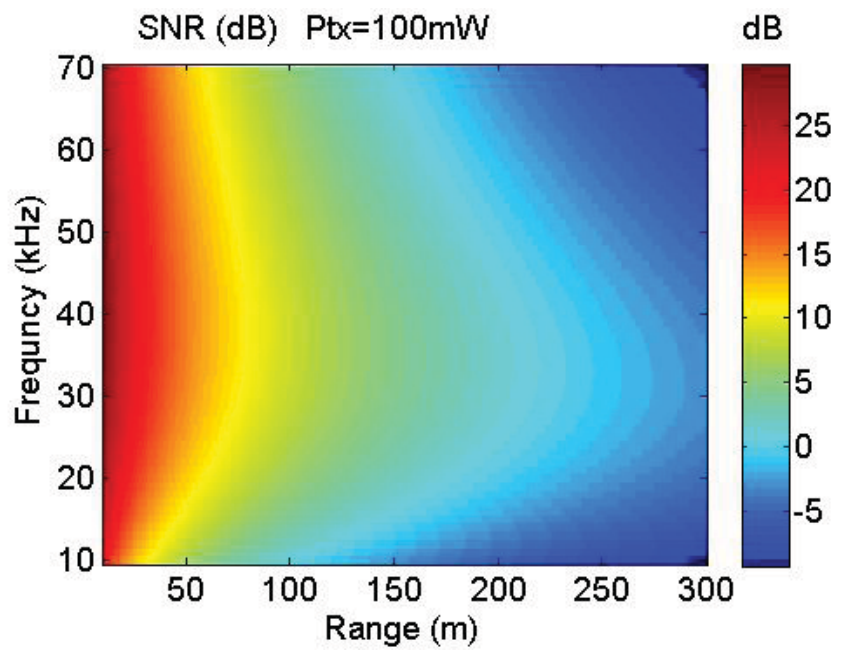

Fig. 5. SNR Ptx $=100 \mathrm{~mW}$

The SNR for various short range transmission scenarios has been evaluated using MATLAB, and illustrate the expected range and frequency dependences. Figure 4 to 6 show that there is an optimal frequency that changes with range. Figure 4 and 5 compares the affect on SNR with changing $P_{t x}$ and shows at $300 \mathrm{~m}$, the SNR reduces from $0 \mathrm{~dB}$ to $-10 \mathrm{~dB}$ as the $P_{t x}$ reduces from $100 \mathrm{~mW}$ to $10 \mathrm{~mW}$ at $30 \mathrm{kHz}$ which is around optimal. Practical underwater acoustic modems vary in their sensitivity with some able to retrieve a signal at $-3 \mathrm{~dB}$ but 
would be unable at $-10 \mathrm{~dB}$. As referenced earlier, variations in $P_{t x}$ need to consider the trade-off between benefits gained from signal strength against noise and fading, and will be included in later work.

\section{B. Frequency Dependent Component of SNR}

As maximising throughput is a major aim for this work, utilising the full capacity of the channel is essential and therefore bandwidth utilisation and optimising SNR are critical factors. Thus, determining the best carrier frequency is critical in this process. This section focuses on choosing the optimum carrier frequency based on the optimal SNR and then establishes bandwidth potential.

The frequency dependent portion of the SNR is the $\mathrm{TL}(\mathrm{r}, \mathrm{f}, \mathrm{d}, \mathrm{t}) \mathrm{N}(\mathrm{f}, \mathrm{w}, \mathrm{s})$ product. Since $\mathrm{SNR}$ is inversely proportional to $T L(r, f, d, t) \sum N(f, w, s)$ factor, the $1 / \mathrm{TL}(\mathrm{r}, \mathrm{f}, \mathrm{d}, \mathrm{t}) \mathrm{N}(\mathrm{f}, \mathrm{w}, \mathrm{s})$ term is used to find the maximum value of frequency for range and is illustrated in Figure 6. Several authors have presented this for longer range, above $500 \mathrm{~m}$ [25], [26], [27]. The two absorption models (Section IIC) present similar responses and optimum frequencies (with minor variations) under similar conditions. Figure 6 does demonstrate that for a particular range (say $100 \mathrm{~m}$ ) there is a dramatic variation in the response curve when the wind state is changed from 0 to $2 \mathrm{~m} / \mathrm{s}$. This is a result of SNR decreasing as the Noise term increases with increasing wind. As frequency increases, the SNR increases as the ratio between the increasing TL and decreasing Noise occurs as frequency increases. As range increases above $700 \mathrm{~m}$, not shown here, the absorption term becomes more significant, reducing the affect of the Noise term and therefore the wind parameter. Shipping variations are not included in these figures as its effect is minor in the frequency range above $10 \mathrm{kHz}$.

As the range increases there is an increasing deviation between the models. The Fisher and Simmons model provides higher optimum frequencies due to the inclusion of the relaxation frequency of boric acid and magnesium sulphate. This variation is expected to occur due to the increasing significance of the TL term compared to the Noise term (which is not effected by range) as range increases.

\section{Channel Bandwidth}

To determine system bandwidth a heuristic of $3 \mathrm{~dB}$ around the optimum frequency has been used [10], [27], [28]. Following a similar method to [28] the bandwidth will be determined $\pm 3 \mathrm{~dB}$ around the optimum frequency $f_{o}(r, d, t)$ which has been chosen as the center frequency. Such that $f_{\min }(r, d, t)$ is the frequency when $\left.T L\left(r, d, t, f_{o}(r, d, t)\right) N\left(f_{o}(r, d, t)\right)-T L(r, d, t, f)\right) N(f) \geq 3$ holds true and similarly for $f_{\max }(r, d, t)$ when $T L(r, d, t, f)) N(f)-T L\left(r, d, t, f_{o}(r, d, t)\right) N\left(f_{o}(r, d, t)\right) \geq 3$ is true. The system bandwidth $\mathrm{B}(\mathrm{r}, \mathrm{d}, \mathrm{t})$ is therefore determined by:

$$
B(r, d, t)=f_{\max }(r, d, t)-f_{\min }(r, d, t)
$$

Thus, for a given range, there exists an optimal frequency from which a range dependent $3 \mathrm{~dB}$ bandwidth can be determined as illustrated in Figure 6. Both the optimal carrier frequency and the $3 \mathrm{~dB}$ system bandwidth decreases as range increases. The higher ranges from $500 \mathrm{~m}$ is included here to correlate values obtained by Chen [27]. Changing $\mathrm{k}$, t, $\mathrm{d}$, and the two absorption models did change the optimum frequency and bandwidth by a minimal amount at short range. Despite this, these parameters should not necessarily be ignored as even between the two models there are a consistent 2-3 $\mathrm{kHz}$ centre frequency difference and a $2 \mathrm{kHz}$ difference in bandwidth with ranges up to $1 \mathrm{~km}$.

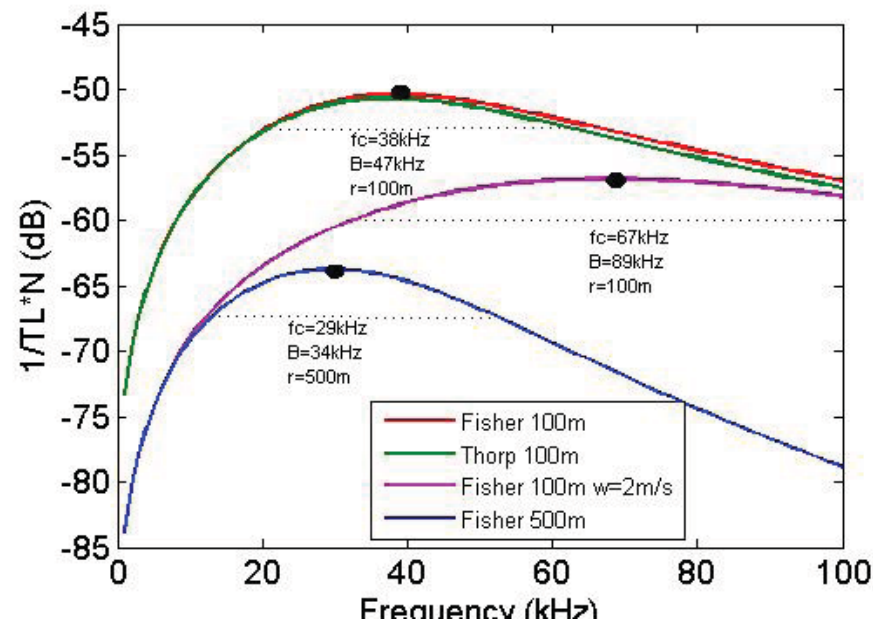

Fig. 6. 3dB Bandwidth

The major changes affecting the $3 \mathrm{~dB}$ channel bandwidth are due to changes in range between transmitter and receiver and with wind variations. Range variations may have been more expected as it is a major parameter in the numerator of the SNR, where optimal frequency and bandwidth decreases as range increases. The wind parameter is interesting as it plays its part in dramatically changing the optimal centre frequency especially at lower ranges and with it the bandwidth. At lower ranges of $50 \mathrm{~m}$, the optimal frequency change for a wind condition change from 0 to $2 \mathrm{~m} / \mathrm{s}$ was almost double, from 40 to $72 \mathrm{kHz}$ whereas at $1 \mathrm{~km}$ there was virtually no frequency change. This translated to a more than doubling of the bandwidth at low ranges.

\section{Short Range Acoustic Channel Challenges}

Thus, the challenges that are imposed by an underwater acoustic channel which will have an impact on the MAC design include:

- the low propagation speed which results in high propagation delay that will directly affect time synchronisation and localisation. This in turn can degrade a MAC's collision detection/avoidance functionality and therefore ultimately reduce throughput. At shorter distances the variability of the speed due to temperature, depth and salinity are significantly lower than at longer ranges and 
thus a MAC protocol can focus on the high delay rather than both high and variable delays,

- a high bit error rate is expected due to the significant multi-path propagation and fading that any acoustic communication underwater will be required to work with. Multi-path and fading are range dependent which may provide some advantages for shorter range communication. In any case the MAC protocol design will need to respond to these channel conditions to ensure conductivity while conserving power,

- limitations due to reliance on battery power that, due to underwater operation is difficult to recharge or replace. For shorter ranges there will be some advantages as the attenuation due to propagation losses would be expected to be less significant, however as shown in Figure 1 this does not appear to change significantly until after approximately $1 \mathrm{~km}$ due to the dominance of the spreading factor. If this is the case then Directivity Index (DI) will play a more significant role at short range and the MAC protocol will need to take this into account. Energy conserving methods will remain critical at any range and thus the MAC design will need to incorporate these, and

- limited bandwidth resulting from a low optimal carrier frequency which will severely reduce the available data rates. As seen in Figure 6 these are dependent on range and at shorter ranges there is a higher optimal carrier frequency and bandwidth which is beneficial, however, these values are still significantly small in comparison to RF.

\section{RATE-RANGE CHARACTERISTICS}

The electro-acoustic interface in the transmitter and receiver are modelled to complete the insight into the limitations of the physical layer system and to define design criteria for MAC protocols. For the short range communication required here, establishing the Probability of Bit Error (BER) and the relationship between the effective data rate and range can identify critical parameters so that within MAC design, optimal network throughput can be achieved while minimising energy consumption in each vehicle.

\section{A. Probability of Bit Error}

Given the acoustic channel characteristics and model developed in Section II and III, the probability of bit error, which is well known for FSK and QPSK modulation techniques [29], can be calculated under varying channel parameters. Firstly, the $\frac{E_{b}}{N_{o}}$ can be found from the SNR by:

$$
\frac{E_{b}}{N_{o}}=S N R\left(r, f, P_{t x}, w, s, \text { selfnoise }, B\right) \times \frac{B_{c}}{R_{b}}
$$

where $R_{b}$ is the bps and $B_{c}$ is the channel bandwidth. Equation 10 and 11 are the uncoded BER for BPSK/QPSK and FSKrespectively:

$$
Q P S K: \quad \mathrm{BER}=\frac{1}{2} \operatorname{erfc}\left[\frac{E_{b}}{N_{o}}\right]^{1 / 2}
$$

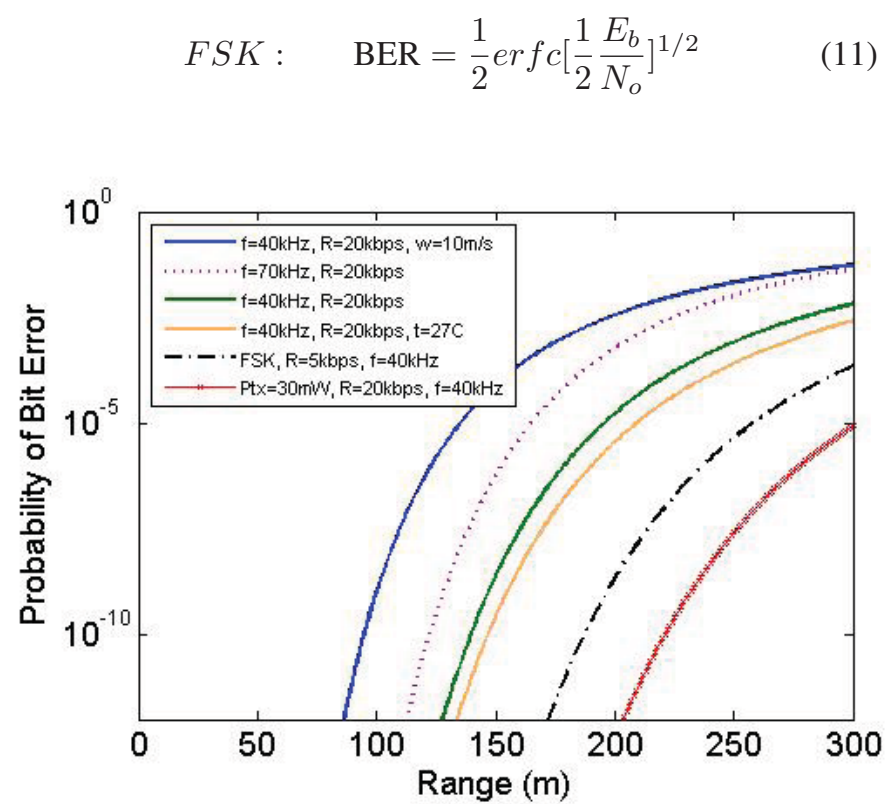

Fig. 7. BER vs Range

Figure 7 shows the BER vs range at a fixed bandwidth of $10 \mathrm{kHz}$ for variations in channel parameters using QPSK and a comparison with FSK modulation. A change in transmitter power $P_{t x}$ from $10 \mathrm{~mW}$ (green) to $30 \mathrm{~mW}$ (red) illustrates the reductions in bit error rate with other parameters the same. The remaining curves use $P_{t x}=10 \mathrm{~mW}$. An increase in the temperature (depth not shown as not found significant) also reduces the bit error probability. On the other hand increases in frequency and wind factor increases the probability of bit error. Alternatively, if the BER is set at $10^{-5}$ then with an increase in transmitter power from 10 to $30 \mathrm{~mW}$ the range increases by approximately $175 \mathrm{~m}$ or if the wind factor reduces from $10 \mathrm{~m} / \mathrm{s}$ to 0 the range increases by approximately $50 \mathrm{~m}$.

\section{B. Rate-Range}

The effective data rate $R_{e f f}$ is determined from the maximum frame transmission rate $F T R_{\max }$ and the frame error rate (FER). Using the probability of bit error found in the last section and the frame size $\mathrm{L}$, the $F E R=1-(1-B E R)^{L}$. This gives the actual frame transmission rate $F T R=1-F E R$ such that the effective data rate can then be calculated by:

$$
R_{e f f}=\left(F T R * F T R_{\max }\right) * L
$$

Figure 8 illustrate the effective bit rate expected under the variations that were explored in Figure 7, while Figure 9 determines the extent of change on the effective bit rate due to changes in packet size. Modifying the transmitter power appears to be a good control of error rates in the channel but the trade-off with the energy consumption will be an issue. Changing frequency bands in response to changing channel conditions can also improve performance as was shown for FSK working at longer ranges by Parrish [30] but a tradeoff with complexity of hardware and software in the acoustic 
transducer will need to be considered. There are minor changes in range for error free transmission with increases in depth but temperature changes can change the error free range by up to $25 \mathrm{~m}$. It is clear to see at the bit level the range dependency on frequency, wind, and the various absorption coefficient parameters for short range underwater acoustic communication systems. Figure 9, shows the change in effective bit rate based on changes in packet size that is an important parameter in MAC design. The packet sizes used here are based on a proposed adaptive token polling protocol previously developed for use in a swarm of AUV [31].

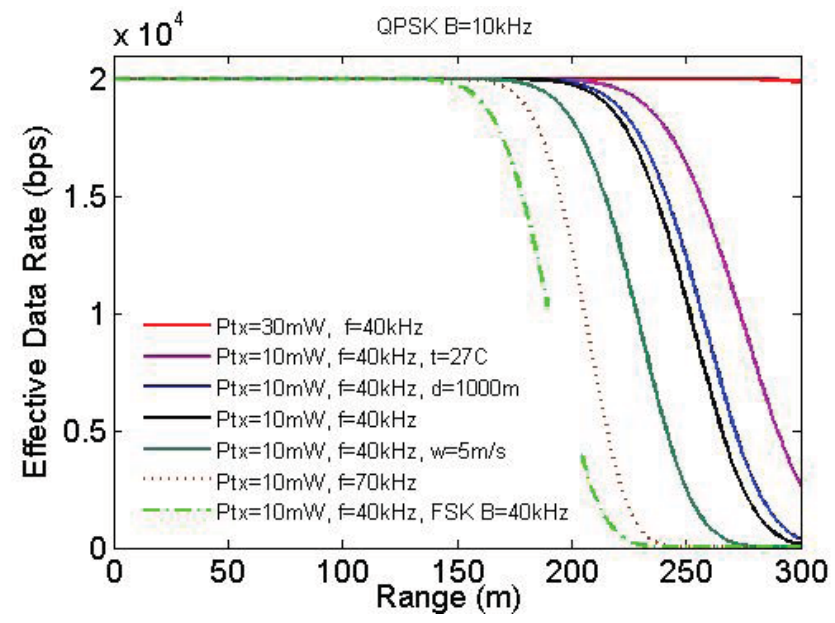

Fig. 8. Effective Data Rate QPSK (bps)

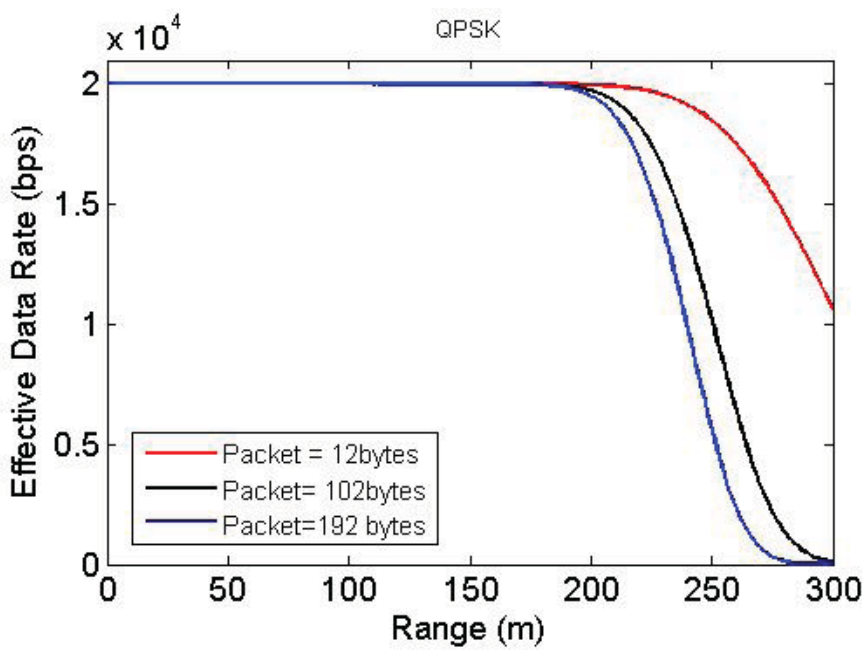

Fig. 9. Effective Data Rate for varying packet size

\section{MAC Design Criteria}

For acoustic transmission underwater, latency and bandwidth limitations are significant design parameters imposed on the MAC layer. For short range communication amongst a swarm of autonomous vehicles, the significant metrics identified for the acoustic communication channel at short range have been again identified in both the probability of bit error and rate range calculations. Transmitter signal power is particularly important due to the significant improvement in effective data rates and BER for higher ranges and the wind component of the noise parameter has a major impact on the probability of bit error. The sensitivity of carrier frequencies on the BER and data rates can be seen in Figures 7 and 8. More specifically for MAC design is the packet size question and for the parameters being investigated here a shorter packet length is seen to improve effective data rates. These predictions for short range communication have been based on acoustic transmission modelling for longer range (far field) communication and thus testing of these criteria experimentally is in progress.

\section{CONCLUSION}

Short range underwater acoustic communication is beginning to emerge as an important dimension in the technological developments underwater as both multi-hop network communication as well as communication between vehicles in a swarm of mobile AUV's is possible. This paper has presented and analysed the sonar equations and underwater acoustic channel specifically for short range communication of $300 \mathrm{~m}$ or less. It has also looked at the effect of these channel parameters on the probability of bit error and effective data rates.

There are some interesting comparisons found with results seen for longer range systems. These insights will be essential for improving the design of MAC protocols for short range underwater communication to ensure the utilisation of the full capacity of the channel. There is still further analysis to be done on the time-variability of the acoustic channel at shorter ranges to determine more appropriate ways to deal with the dynamics associated with the time and spacial variations expected when operating in a swarm.

Experimental testing is being set up to validate the conclusions above and this will be presented in future work.

\section{ACKNOWLEDGEMENT}

The authors would like to thank ATSA Defence Services for their support of this research project.

\section{REFERENCES}

[1] M. Dunbabin, J. Roberts, K. Usher, G. Winstanley, and P. Corke, "A hybrid auv design for shallow water reef navigation," in Proc. International Conference on Robotics and Automation (ICRA), April 2005, pp. 2117-2122.

[2] J. Holmes and J. Lynch, "An autonomous underwater vehicle towed array for ocean acoustic measurements and inversion," The Journal of the Acoustical Society of America, 2005.

[3] P. B. Raja Jurdak, Cristina Videira Lopes, "A software acoustic modems for short range mote-based underwater sensor networks," IEEE, 2006.

[4] F. Salva-Garau and M. Stojanovic, "Multi-cluster protocol for ad hoc mobile underwater acoustic networks," in OCEANS 2003. Proceedings, vol. 1, 2003, pp. 91-98.

[5] C. Benson, M. Ryan, and M. Frater, "On the benefits of high absorption in practical multi-hop networks," in OCEANS 2007 - Europe, 2007, pp. $1-6$.

[6] J. Preisig, "Acoustic propagation considerations for underwater acoustic communications network development," in WUWNet Conference 2006. Workshop on Underwater Networks (WUWNet), 2006. 
[7] F. Fisher and V. Simmons, "Sound absorption in sea water," J. Acoust. Soc. Am., vol. 62, no. 3, 1977.

[8] R. Francois and G. Garrison, "Sound absorption based on ocean measurements: Part 1 and 2," Journal of the Acoustical Society of America, vol. 72, no. 3,6, pp. 896-907, 1879-1890, 1982.

[9] M. Domingo, "Overview of channel models for underwater wireless communication networks," Physical Communication, pp. 163-182, 2008.

[10] A. Sehgal, I. Tumar, and J. Schonwalder, "Variability of available capacity due to the effects of depth and temperature in the underwater acoustic communication channel,' in IEEE OCEANS '09., May 2009, pp. 1-6.

[11] A. Waite, Sonar for Practicing Engineers, 3rd ed. Wiley, 2002.

[12] M. Stojanovic, "Underwater wireless communication," Tutorial OCEANS'10, IEEE, May 2010, Sydney.

[13] R. Urick, Principles of Underwater Sound for Engineers. McGraw-Hill, 1967.

[14] J. Caruthers, Fundamentals of Marine Acoustics. Elsevier Scientific Publishing, 1977.

[15] L. Kinsler, A. Frey, A. Coppens, and J. Sanders, Fundementals of Acoustics. John Wiley and Sons, 1982.

[16] C. University, Principles of Underwater Sound, C. Eckart, Ed. Research Analysis Group, National Research Council, 1952.

[17] A. Essebbar, G. Loubet, and F. Vial, "Underwater acoustic channel simulations for communication," in OCEANS '94. 'Oceans Engineering for Today's Technology and Tomorrow's Preservation.' Proceedings, vol. 3, September 1994, pp. 495-500.

[18] P. C. Etter, Underwater Acoustic Modeling and Simulation, 3rd ed. Spon Press, 2003.

[19] R. Coates, Underwater Acoustic Systems. John Wiley and Sons, 1989.

[20] M. Chitre, "A high-frequency warm shallow water acoustic communications channel model and measurements," Journal of the Acoustical Society of America, vol. 122, no. 5, pp. 2580-2586, 2007. [Online]. Available: www.scopus.com

[21] A. Radosevic, J. Proakis, and M. Stojanovic, "Statistical characterization and capacity of shallow water acoustic channels," in IEEE OCEANS '09., 2009, pp. 1-8

[22] S.-M. Kim, S.-H. Byun, S.-G. Kim, and Y.-K. Lim, "Experimental analysis of statistical properties of underwater channel in a very shallow water using narrow and broadband signals," in OCEANS 2009, MTS/IEEE Biloxi - Marine Technology for Our Future: Global and Local Challenges, 2009, pp. 1-4.

[23] J. T. Hanson, "Link budget analysis for underwater acoustic signalling," Master's thesis, Naval Postgraduate School, Monterey, California, 2002.

[24] T. Stabile, A. Zollo, M. Vassallo, and G. Iannaccone, "Underwater acoustic channel properties in the gulf of naples and their effects on digital data transmission," Annals of Geophysics, vol. 50, no. 3, June 2007.

[25] N. Nasri, A. Kachouri, L. Andrieux, and M. Samet, "Design considerations for wireless underwater communication transceiver," in International COnference on Signals, Circuits and Systems, 2008.

[26] M. Stojanovic, "On the relationship between capcity and distance in an underwater acoustic communication channel," in International Workshop on Underwater Networks, WUWNet'06. ACM, 2006.

[27] W. Chen and U. Mitra, "Packet scheduling for multihopped underwater acoustic communication networks," in IEEE OCEANS'07, October 2007, pp. 1-6.

[28] M. Stojanovic and L. Freitag, "Multichannel detection for wideband underwater acoustic cdma communications," IEEE Journal of Oceanic Engineering, vol. 31, no. 3, pp. 685-695, 2006.

[29] T. Rappaport, Wireless Communications, Principles and Practice. Prentice Hall, 1996.

[30] N. Parrish, S. Roy, W. Fox, and P. Arabshahi, "Rate-range for an fh-fsk acoustic modem," in WuWNet '07: Proceedings of the second workshop on Underwater networks. New York, NY, USA: ACM, 2007, pp. 93-96.

[31] G. Burrowes and J. Khan, "Adaptive token polling mac protocol for wireless underwater networks," IEEE International Symposium on Wireless and Pervasive Computing, 2009. 